\title{
CTA sensitivity to the high-energy tail of the Fermi GeV excess
}

\author{
Oscar Macias, ${ }^{a, b, *}$ Harm van Leijen, ${ }^{a, b}$ Deheng Song, ${ }^{c}$ Shin'ichiro Ando, ${ }^{b, a}$ \\ Shunsaku Horiuchi ${ }^{c}$ and Roland M. Crocker ${ }^{d}$ \\ ${ }^{a}$ Kavli Institute for the Physics and Mathematics of the Universe (WPI), University of Tokyo, \\ Kashiwa, Chiba 277-8583, Japan \\ ${ }^{b}$ GRAPPA Institute, Institute of Physics, University of Amsterdam, \\ 1098 XH Amsterdam, The Netherlands \\ ${ }^{c}$ Center for Neutrino Physics, Department of Physics, Virginia Tech, \\ VA 24061, Blacksburg, USA \\ ${ }^{d}$ Research School of Astronomy and Astrophysics, Australian National University, \\ ACT 2611, Canberra, Australia \\ E-mail: o.a.maciasramirez@uva.nl
}

Past studies of the Fermi Galactic Center Excess (GCE) have found evidence for a "high-energy tail" in the GCE spectrum. Such a signature could be the result of inverse-Compton (IC) gamma rays produced by the injection of electrons/positrons into the interstellar medium by the putative population of millisecond pulsar (MSPs) responsible for the GCE. For this ICRC 2021 contribution, we present the results of an exhaustive study on simulated data, in which we analyze the detection potential of the forthcoming Cherenkov Telescope Array (CTA) to the high-energy tail of the Fermi $\mathrm{GeV}$ excess. In particular, we find that CTA will have sufficient sensitivity to detect this signal for physically reasonable electron/positron acceleration efficiencies, provided that the Galactic diffuse emission model (GDE) is well understood. Furthermore, we discuss the necessary conditions for a reliable CTA discovery in the case of a high degree of uncertainties in the GDE model. We also show that in the event that CTA observes an excess of diffuse gamma rays in the Galactic bulge, it will be able to discriminate between the dark matter and MSPs hypotheses, based on their distinct spatial morphologies.

$37^{\text {th }}$ International Cosmic Ray Conference (ICRC 2021)

July 12 th - 23rd, 2021

Online - Berlin, Germany

\footnotetext{
*Presenter
} 


\section{Introduction}

Fermi-LAT observations of the inner $\sim 10^{\circ}$ of the Galactic center (GC) have revealed a highly statistically significant $\gamma$-ray excess (see e.g., [2, 5, 10, 11], and references therein). Though the origins of this Galactic Center Excess (GCE) are largely unknown, the current consensus is that it is not due to a fluctuation of the astrophysical background. Interestingly, the most highly debated hypotheses for the GCE are: a large unresolved-population of Galactic bulge millisecond pulsars (MSPs), and dark matter (DM) particles [with masses $\sim 30-60 \mathrm{GeV}$ self-annihilating in $b$-quarks] spatially distributed as a Navarro-Frenk-White (NFW) profile with a mild slope $(\gamma \simeq 1.2)$.

Given that MSPs can emit a spectrum that is very similar to that of DM annihilations, both the DM and MSPs explanations appeared to be completely degenerate in earlier studies. However, recent analyses have shown that two independent characteristics of the signal could be crucial to discriminate between the two main hypotheses for the GCE: (i) the photon count statistics [7, 13], and (ii) the spatial morphology of the signal $[8,15]$. Interestingly, several independent studies $[1$, $8,9,15,16]$ have demonstrated that the spatial morphology of the GCE is correlated with stellar mass in the Galactic bulge; a result that strongly supports the MSPs hypothesis for the GCE, and is completely separate of conclusions drawn from the photon count statistics studies.

The next few years are likely to see increased efforts in observing the predicted multi-wavelength signatures of the putative MSPs responsible for the GCE. A method that is particularly promising and is the focus of this ICRC 2021 contribution-is to look for a TeV-scale inverse Compton (IC) signal produced by the injection of very-high-energy $e^{ \pm}$into the interstellar medium. The theoretical and observational motivations for this search channel are discussed at length in Refs. [17, 20]. In particular, previous analyses (e.g., [14]) of the GCE have claimed the presence of a high-energy tail in the GCE spectrum which might well extend to multi-TeV energies. Here, we present the results a realistic assessment of the capabilities of the forthcomming Cherenkov Telescope Array (CTA) to an unresolved population of MSPs in the GC. We use simulated data to consider scenarios where the Galactic diffuse emission (GDE) is perfectly known, and others where the GDE is mismodeled. In this proceedings, we summarize our modeling assumptions, and main results. The readers are encouraged to see Ref. [17] for further details.

\section{Analysis pipeline}

\subsection{Data selection}

We assumed 500 hours of CTA observations from the inner $10^{\circ} \times 10^{\circ}$ around the GC, and selected (simulated) events in the energy range $16 \mathrm{GeV}-158 \mathrm{TeV}$. The mock data was created by first performing a convolution of our GDE/signal models with the publicly-available ${ }^{1}$ instrument response function (IRF) CTA-Performanceprod-3bv1-South-20deg -average-50h. root3 and then taking Poissonian draws from these. Additionally, we masked the region of the Galactic plane given by $|b| \leq 0.3^{\circ}$, as well as all the point sources in the 3FHL [6] that have a hard spectrum (this is similar to Ref. [19]). Furthermore, we divided the data in 11 logarithmically spaced energy bins, and for the spatial bins we used $0.5^{\circ} \times 0.5^{\circ}$. Note that the spatial bins are given by the minimum angular resolution of our gas maps.

\footnotetext{
1https://www.cta-observatory.org/science/cta-performance/
} 

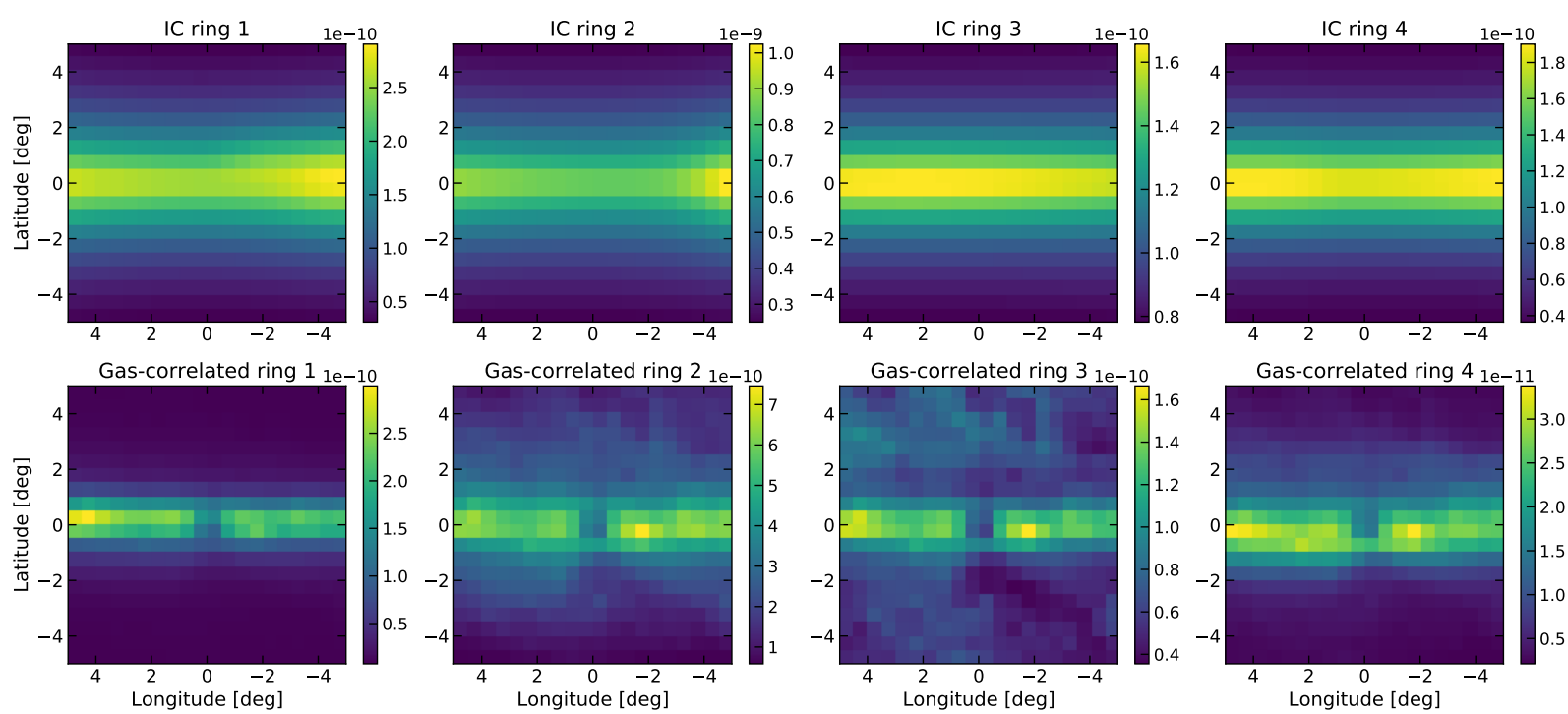

Figure 1: The spatial morphology of the background gas-correlated and inverse Compton components at an energy of $\approx 11 \mathrm{TeV}$. The region of interest corresponds to the inner $10^{\circ} \times 10^{\circ}$ of the GC, and the maps have a resolution of $0.5^{\circ} \times 0.5^{\circ}$, which is given by the spatial resolution of the gas maps. All the maps were computed using GALPROP v56, and were divided in Galactocentric rings of size 0 - 3.5, 3.5 - 8.0, 8.0 - 10.0, and $10.0-50.0 \mathrm{kpc}$.

\subsection{Construction of the GDE model}

We performed simulations of the astrophysical background in the GC by using GALPROP v56 [12, 18]. This is the latest release of the code, which contains numerous improvements with respect to previous versions. Notably, it now includes 3D models for the interstellar radiation fields,
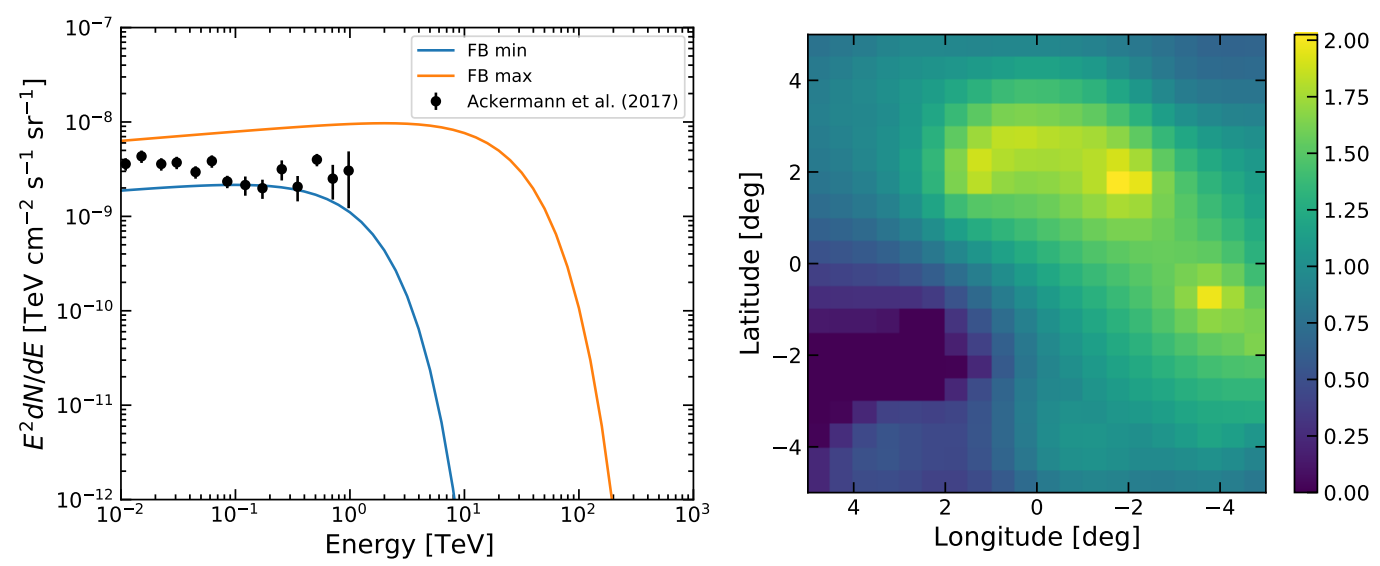

Figure 2: Left: The two different spectral models assumed for the Fermi bubbles (FB). The $\mathrm{FB}_{\min }$ and $\mathrm{FB}_{\max }$ models were selected such that these encompass the range of uncertainties on the measured Fermi spectrum [4] (black points), and they do not overshoot the H.E.S.S Pevatron measurements. Right:The spatial morphology of the FB template in the inner $10^{\circ} \times 10^{\circ}$ of the GC. This map was created in Macias et al. [16] by applying an image restoration algorithm on the map originally constructed in [3]). The color bar is displayed in arbitrary units. 

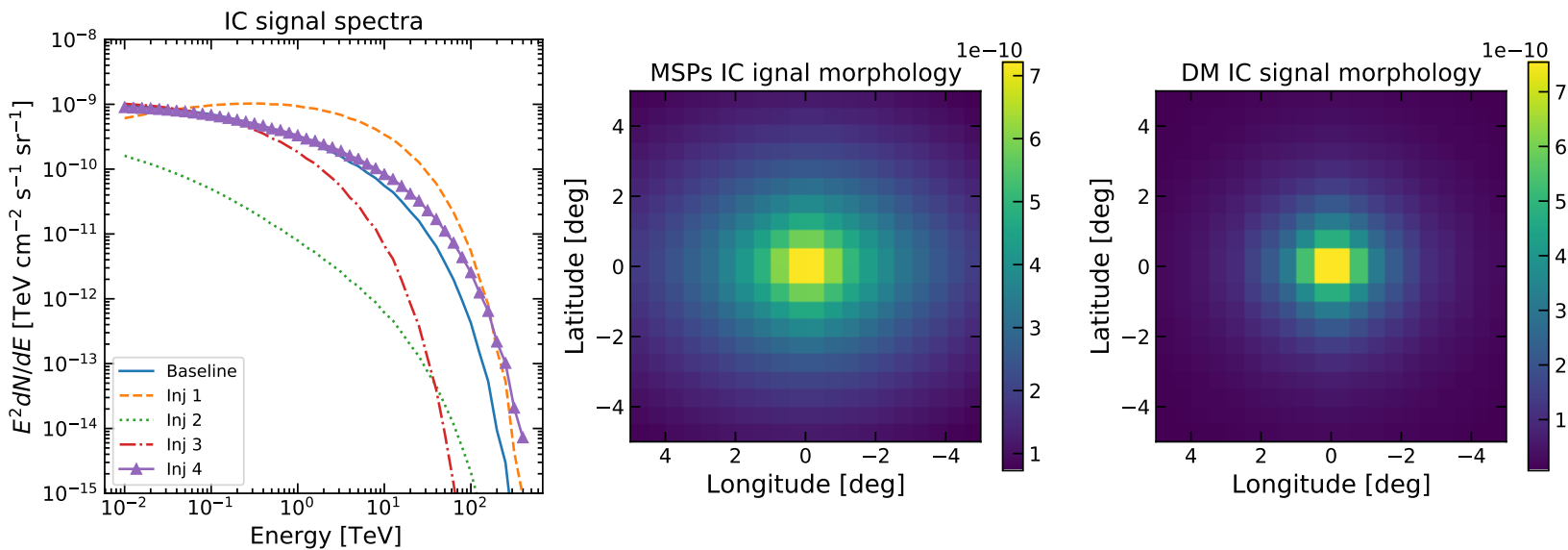

Figure 3: Left: The inverse Compton spectra produced by the putative population of MSPs that explain the GCE. The different spectral models result from various $e^{ \pm}$injection spectra considered in this work (See also Table 2 in Ref. [17]). Middle: The spatial morphology of the IC signal produced by a bulge population of MSPs injecting $e^{ \pm}$with a mean spectrum as given by Inj1 (at $1 \mathrm{TeV}$ ). Right: Same as the middle panel, except that here the MSPs are assumed to follow a spherical distribution as given by an NFW profile with a mild slope $(\gamma=1.2)$.

and interstellar gas-which enables us to abandon assumptions of axisymmetry for the Galaxy. Figure 1 shows our predicted IC maps, and gas-correlated maps at $\sim 11 \mathrm{TeV}$. The normalization of these components have been fitted to Fermi-LAT data, so that these constitute physically motivated predictions of multi-TeV diffuse emission.

We have also included in our analysis templates for the low-latitude Fermi bubbles (FB) (see Fig. 2). As introduced first in [19], we considered two flux models for the FB. Our motivation for this choice is that by repeating our pipeline with these two alternatives, we can estimate the effect that uncertainties in this component has in our results.

\subsection{Signal modeling}

Here, we assume that the GCE is originated either by a new population of MSPs distributed as the stars in the Galactic bulge or DM particles distributed as an NFW squared profile. To investigate the capabilities of CTA to distinguish the IC signals produced by either of these models, we assume that they both have the same $e^{ \pm}$injection spectrum, but different spatial morphologies. Interestingly, for the MSPs scenario, we can write down a relation between the prompt $\gamma$-ray luminosity $\left(L_{\gamma, \text { prompt }}\right)$ and the $e^{ \pm}$luminosity $L_{e^{ \pm}}$. Specifically,

$$
L_{e^{ \pm}}=f_{e^{ \pm}} \dot{E}=\frac{f_{e^{ \pm}}}{f_{\gamma}} L_{\gamma, \mathrm{prompt}} \simeq 10 f_{e^{ \pm}} L_{\gamma, \mathrm{prompt}},
$$

where $(\dot{E})$ is the MSP spin-down rate, $f_{e^{ \pm}}$is the $e^{ \pm}$efficiency, and we have assumed $f_{\gamma}=10 \%$ [21]. This way, we can make physical sense of our results by comparing the $f_{e^{ \pm}}$values required for a CTA discovery with the ones estimated from observations of MSPs elsewhere.

The particular shape of the $e^{ \pm}$injection spectrum is highly uncertain. To account for this, we 

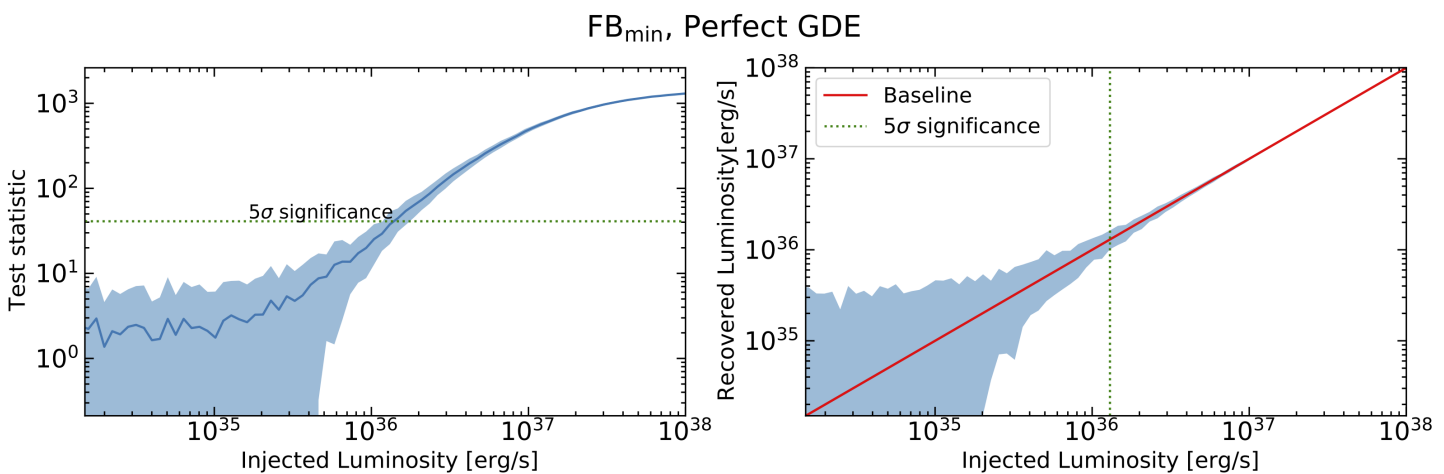

$\mathrm{FB}_{\max }$, mismodeling of the GDE
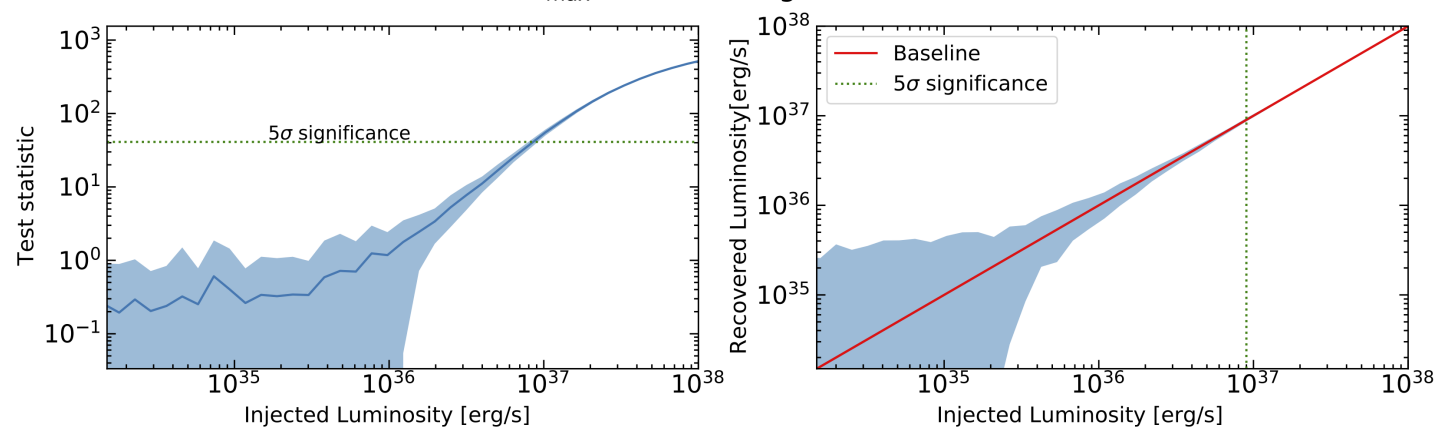

Figure 4: The results of the signal-injection-recovery tests for varying degrees of mismodeling of the GDE model. The top panel assumes perfect knowledge of the GDE and the $\mathrm{FB}_{\text {min }}$ model, while the bottom panels assume mismodeling of the GDE and the $\mathrm{FB}_{\text {max }}$ model (see Fig. 2). All cases displayed correspond to the $e^{ \pm}$injection spectrum Baseline (see Table 2 in Ref. [17]). Left: Test Statistic of the MSPs' IC signal as a function of the IC gamma-ray luminosity. The $5 \sigma$ threshold is presented as a green dotted line. The blue lines show the mean values and the blue shaded regions gives their variance. Right: Comparison of the injected signal vs recovered signal. The red line shows the ideal case in which the extracted signal matches the injected signal perfectly. The blue region shows the recovered IC signals.

used a power-law with an exponential cutoff of the form

$$
\frac{d^{2} N}{d E d t} \propto E^{-\Gamma} \exp \left(-E / E_{\text {cut }}\right)
$$

where $\Gamma$ is the slope, and $E_{\text {cut }}$ is the energy cutoff. We solved the CR transport equation for these $e^{ \pm}$using GAPROP V56 in its 3D configuration. The propagation parameter setup is the same as the SA50 model in Ref. [12]. As can be seen in Fig. 3, we considered several possible values for $\Gamma$, and $E_{\text {cut }}$ (five different models), which cover a substantial range of the expected uncertainties on these parameters. Figure 3 also shows that the spatial morphology of the MSPs and DM induced IC signals are indeed predicted to be different. Further details about our signal modeling procedure can be seen in Ref. [17]. In the following, we set out to investigate the necessary conditions for a robust CTA detection of this IC signal.

\subsection{Analysis Pipeline}

We analyse the simulated CTA using a bin-by-bin analysis method. Specifically, we split the data into 11 energy bins logarithmically separated in the energy range $16 \mathrm{GeV}-158 \mathrm{TeV}$. At each 


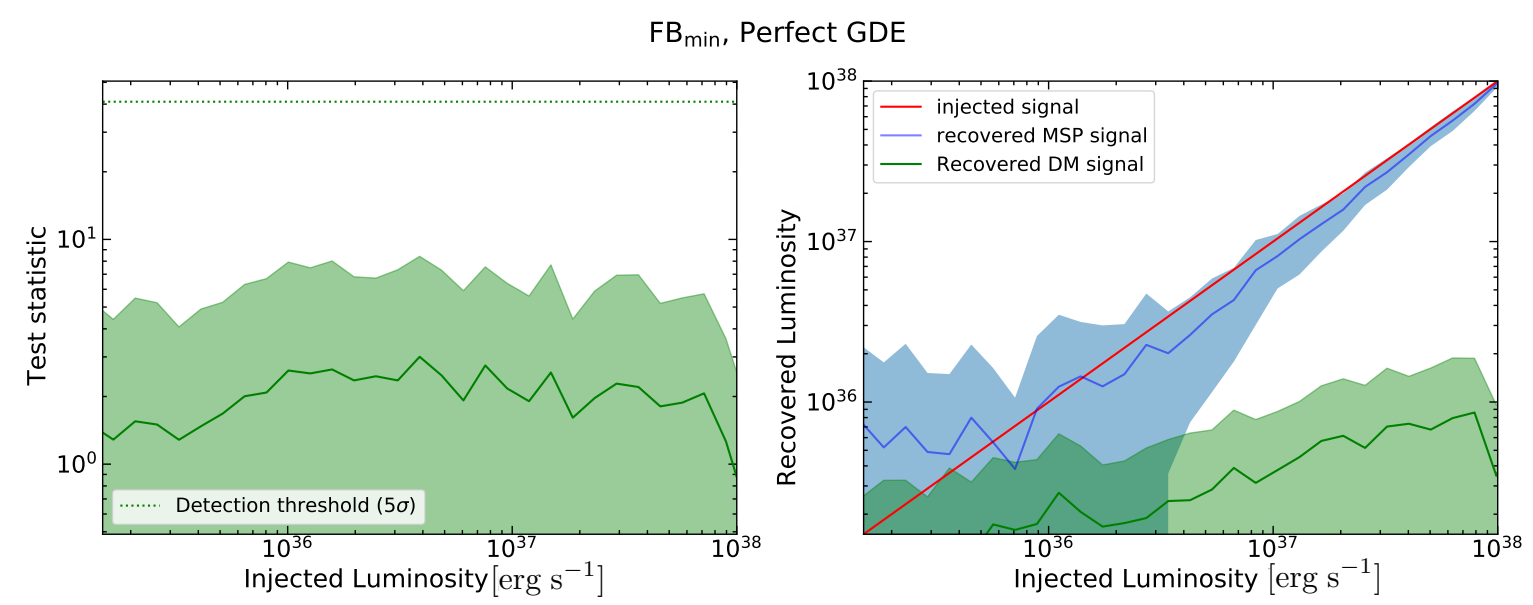

$\mathrm{FB}_{\text {max }}$, mismodelling of the GDE
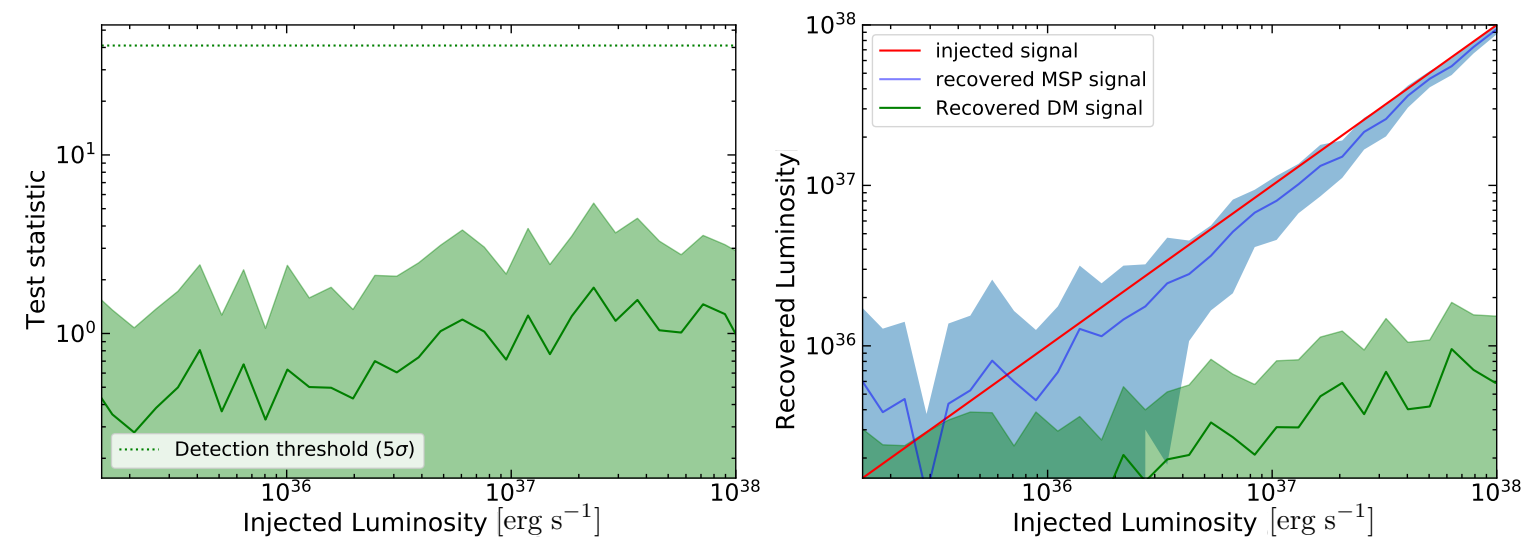

Figure 5: The results of the tests for morphological degenerecies between an IC signal produced by either DM or a bulge population of MSPs. The cases considered are the same as in Fig. 4. The signal injection tests are performed by including a MSPs IC signal into the mock data and subsequently attempting to recover the signal using both a DM and the MSPs IC templates. The left panel shows the TS values for the DM IC template, the right panel shows the actual signal recovery tests results. It is seen that for large enough IC luminosities there is very little MSPs IC flux that is absorved by the DM IC template.

energy bin, we assume that the template spectra is given by $d N / d E=N_{0} E^{-2}$, and use a maximum likelihood routine to obtain the best-fit normalization $N_{0}$. Since the energy bins are relatively small, this method allows us to reconstruct the simulated spectra in model-independent way.

In order to evaluate the impact of uncertainties in the GDE model on our results, we considered two scenarios. (i) Optimistic scenario: In this case, we assume that the astrophysical background is perfectly known, and assume the $\mathrm{FB}_{\text {min }}$, which has the minimum gamma ray intensity that is allowed by Fermi observations of this component. In practice, this means that we fit the data using the same templates that we used generating it in the first place. (ii) Conservative scenario: For this, we generate the simulated data using the GDE model created with GALPROP, and assume the maximum allowed flux model for the $\mathrm{FB}$ ( $\mathrm{FB}_{\max }$ model). However, we fit the data using the hydrodynamical gas maps proposed in [15] (for the gas-correlated component), and use a 3D IC 


\begin{tabular}{c|c|c|c|c}
\hline \hline \multicolumn{5}{c}{ Minimum $f_{e^{ \pm}}$for detection [\%] } \\
\hline Baseline & Inj1 & Inj2 & Inj3 & Inj4 \\
\hline \multicolumn{5}{c}{$\mathrm{FB}_{\min }$, perfect GDE. } \\
\hline $10.5 \%$ & $2.9 \%$ & $158.4 \%$ & $24.3 \%$ & $8.2 \%$ \\
\hline \multicolumn{5}{c}{$\mathrm{FB}_{\max }$, mismodeling of the GDE. } \\
\hline $72.9 \%$ & $51.8 \%$ & $326.7 \%$ & $70.4 \%$ & $74.1 \%$ \\
\hline \hline
\end{tabular}

Table 1: Minimum $e^{ \pm}$injection efficiency $\left(f_{e^{ \pm}}\right)$required for a $5 \sigma$ CTA detection of the Galactic bulge population of MSPs responsible for the GCE (see also Eq. 1). To obtain these efficiencies we calculated the gamma-ray luminosities for energies greater than $700 \mathrm{MeV}$.

map that is not divided in rings-and so is more restrictive during the fits.

\section{Results}

Figure 4 shows the main results of our analysis. We injected signals of different magnitudes into the data, and then used the bin-by-bin procedure to recover the signals. As can be seen, for IC luminosities that are above the detection threshold, the injected signals are adequately recovered by our pipeline. However, when we switch from the optimistic to the conservative scenario, we observe a drop in sensitivity by almost one order of magnitude. Figure 5 shows the results of a similar test, except that this time, we attempted to recover the injected MSPs IC signal by including in the fits both the MSPs IC and DM IC templates (appropriately nested in the fit). Interestingly, our study shows that CTA has the capability to distinguish between the two models, just based on their different morphologies, for luminosities above the detection threshold. In Table 1, we show the minimum $f_{e^{ \pm}}$required for a robust CTA detection of the high-energy tail of the GCE. We note that Inj2 spectral model has the highest degree of degeneracy with GDE model, and therefore is the most difficult to detect. However, given the theoretical and observational uncertainties on $f_{e^{ \pm}}$, CTA might still be able to detect such a signal.

\section{Conclusions}

We have shown that CTA has the necessary sensitivity to detect the high-energy tail of the GCE for physically plausible electron acceleration efficiencies. Though the CTA sensitivity to this signal generally decreases under the assumption of an imperfect GDE model, a CTA detection could still be viable. Furthermore, in the event that CTA observes an excess of diffuse gamma rays in the GC, it will be able to discriminate between the DM and MSPs hypotheses.

\section{References}

[1] Abazajian, K. N., Horiuchi, S., Kaplinghat, M., Keeley, R. E., \& Macias, O. 2020, Phys. Rev. D, 102, 043012, doi: 10.1103/PhysRevD.102.043012

[2] Abazajian, K. N., \& Kaplinghat, M. 2012, Phys. Rev., D86, 083511, doi: 10 . 1103/PhysRevD . 86.083511, 10.1103/PhysRevD.87.129902 
[3] Ackermann, M., et al. 2014, Astrophys. J., 793, 64, doi: 10 . 1088/0004-637X/793/1/64

[4] —. 2017, Astrophys. J., 840, 43, doi: 10.3847/1538-4357/aa6cab

[5] Ajello, M., et al. 2016, Astrophys. J., 819, 44, doi: 10 . 3847/0004-637X/819/1/44

[6] —. 2017, Astrophys. J. Suppl., 232, 18, doi: 10.3847/1538-4365/aa8221

[7] Bartels, R., Krishnamurthy, S., \& Weniger, C. 2016, Phys. Rev. Lett., 116, 051102, doi: 10. 1103/PhysRevLett. 116.051102

[8] Bartels, R., Storm, E., Weniger, C., \& Calore, F. 2018, Nat. Astron., 2, 819, doi: 10.1038/ s41550-018-0531-z

[9] Calore, F., Donato, F., \& Manconi, S. 2021, arXiv e-prints, arXiv:2102.12497. https: //arxiv.org/abs/2102.12497

[10] Gordon, C., \& Macias, O. 2013, Phys. Rev., D88, 083521, doi: 10.1103/PhysRevD.88. 083521, 10.1103/PhysRevD.89.049901

[11] Hooper, D., \& Goodenough, L. 2011, Phys. Lett., B697, 412, doi: 10.1016/j . physletb. 2011.02 .029

[12] Jóhannesson, G. 1., Porter, T. A., \& Moskalenko, I. V. 2018, Astrophys. J., 856, 45, doi: 10. 3847/1538-4357/aab26e

[13] Lee, S. K., Lisanti, M., Safdi, B. R., Slatyer, T. R., \& Xue, W. 2016, Phys. Rev. Lett., 116, 051103, doi: 10.1103/PhysRevLett.116.051103

[14] Linden, T., Rodd, N. L., Safdi, B. R., \& Slatyer, T. R. 2016, Phys. Rev. D, 94, 103013, doi: 10.1103/PhysRevD.94.103013

[15] Macias, O., Gordon, C., Crocker, R. M., et al. 2018, Nat. Astron., 2, 387, doi: 10.1038/ s41550-018-0414-3

[16] Macias, O., Horiuchi, S., Kaplinghat, M., et al. 2019, JCAP, 09, 042, doi: 10.1088/ $1475-7516 / 2019 / 09 / 042$

[17] Macias, O., van Leijen, H., Song, D., et al. 2021, doi: 10 . 1093/mnras/stab1450

[18] Porter, T. A., Jóhannesson, G., \& Moskalenko, I. V. 2017, Astrophys. J., 846, 67, doi: 10 . $3847 / 1538-4357 /$ aa844d

[19] Rinchiuso, L., Macias, O., Moulin, E., Rodd, N. L., \& Slatyer, T. R. 2021, Phys. Rev. D, 103, 023011, doi: 10.1103/PhysRevD. 103.023011

[20] Song, D., Macias, O., \& Horiuchi, S. 2019, Phys. Rev., D99, 123020, doi: 10.1103/ PhysRevD. 99.123020

[21] Song, D., Macias, O., Horiuchi, S., Crocker, R. M., \& Nataf, D. M. 2021, arXiv e-prints, arXiv:2102.00061. https://arxiv.org/abs/2102.00061 